\title{
PERSISTING HYPOTROPIAS FOLLOWING PROTECTIVE PTOSIS INDUCED BY BOTULINUM NEUROTOXIN
}

\author{
P. L. O. HEYWORTH and J. P. LEE \\ London
}

\begin{abstract}
SUMMARY
Botulinum toxin A induced ptosis (BTXAP) has become an established method for producing a temporary ptosis for corneal protection. Adams et al ${ }^{1}$ reported from an initial series of 15 patients and observed that the ptosis lasted for a mean period of 2.5 weeks and that full recovery was achieved after a mean of 8.1 weeks. They noted that in $80 \%$ of cases there was a temporary superior rectus weakness which lasted for a mean of 6 weeks. We present three cases in which the superior rectus weakness was permanent and required corrective strabismus surgery. We believe that these are the first cases reported. We propose two possible mechanisms which may be acting together: firstly that a prolonged period of occlusion may have led to a breakdown of fusion, and secondly that following botulinum toxin induced superior rectus weakness there was contracture of the ipsilateral antagonist muscle further disrupting fusional mechanisms.
\end{abstract}

\section{METHODS}

Botulinum toxin A induced protective ptosis (BTXAP) is achieved by giving an injection using a 25 gauge $25 \mathrm{~mm}$ needle using a solution containing $62.5 \mathrm{pg}(2.5 \mathrm{U})$ of botulinum toxin A per $0.1 \mathrm{ml}$. This is the same concentration as for treating strabismus. The needle is directed along the roof of the orbit at a point midway between the medial and lateral walls with the eye depressed. A $0.3 \mathrm{ml}$ bleb containing $187.5 \mathrm{pg}(7.5 \mathrm{U})$ is injected just beneath the roof of the orbit with the needle advanced to its full extent. The total dose is three times the standard dose for treating strabismus. The bleb is left to diffuse over the levator complex. The technique is performed by 'feel' and no electromyographic control is required. The effect is not instantaneous, taking some days to develop. During this time it is necessary to continue taping and medical therapy.

Correspondence to: P. L. O. Heyworth, Moorfields Eye Hospital, City Road, London EC1V 2PD, UK.

Eye (1994) 8, 511-515 $\quad$ C 1994 Royal College of Ophthalmologists

\section{CASE REPORTS}

\section{Case 1}

A 53-year-old woman had a pleomorphic adenoma of the lacrimal gland removed via a left-sided lateral orbitotomy. She was recorded as having full ocular movements before and 1 month following surgery. As a result of a dry eye and lagophthalmos due to seventh nerve paresis she developed an exposure keratitis. She received two doses of botulinum toxin within a month. The ptosis was complete; however, 1 month later she complained of diplopia and 7 months following the surgery she was recorded as having a 26 dioptre hypotropia in the primary position. The Hess chart is shown in Fig. 1. The degree of concomitancy may suggest that a long-standing deviation was present before the onset of symptoms. A traction test at this time showed mild restriction of elevation.

Initially the patient was treated with a standard dose of botulinum toxin to the left inferior rectus muscle. This temporarily restored binocular vision to all positions of gaze (Fig. 2). Within a month the diplopia had returned and persisted for 2 years before she underwent an adjustable inferior rectus recession. Binocular single vision was restored (Fig. 3) to all positions of gaze and she has been discharged from our care.

\section{Case 2}

A 58-year-old woman developed exposure keratitis following a right-sided Bell's palsy. She was treated with two doses of botulinum toxin within a month.

One month following the last injection she noted diplopia on downgaze which became apparent in all positions of gaze as the ptosis resolved. Thirteen months later she was recorded as having an intermittent 25 dioptre hypotropia in the primary position with a marked superior oblique overaction (Fig. 4). Two years following the onset of diplopia she underwent a Knapp procedure following a negative traction test. This restored binocular single vision 

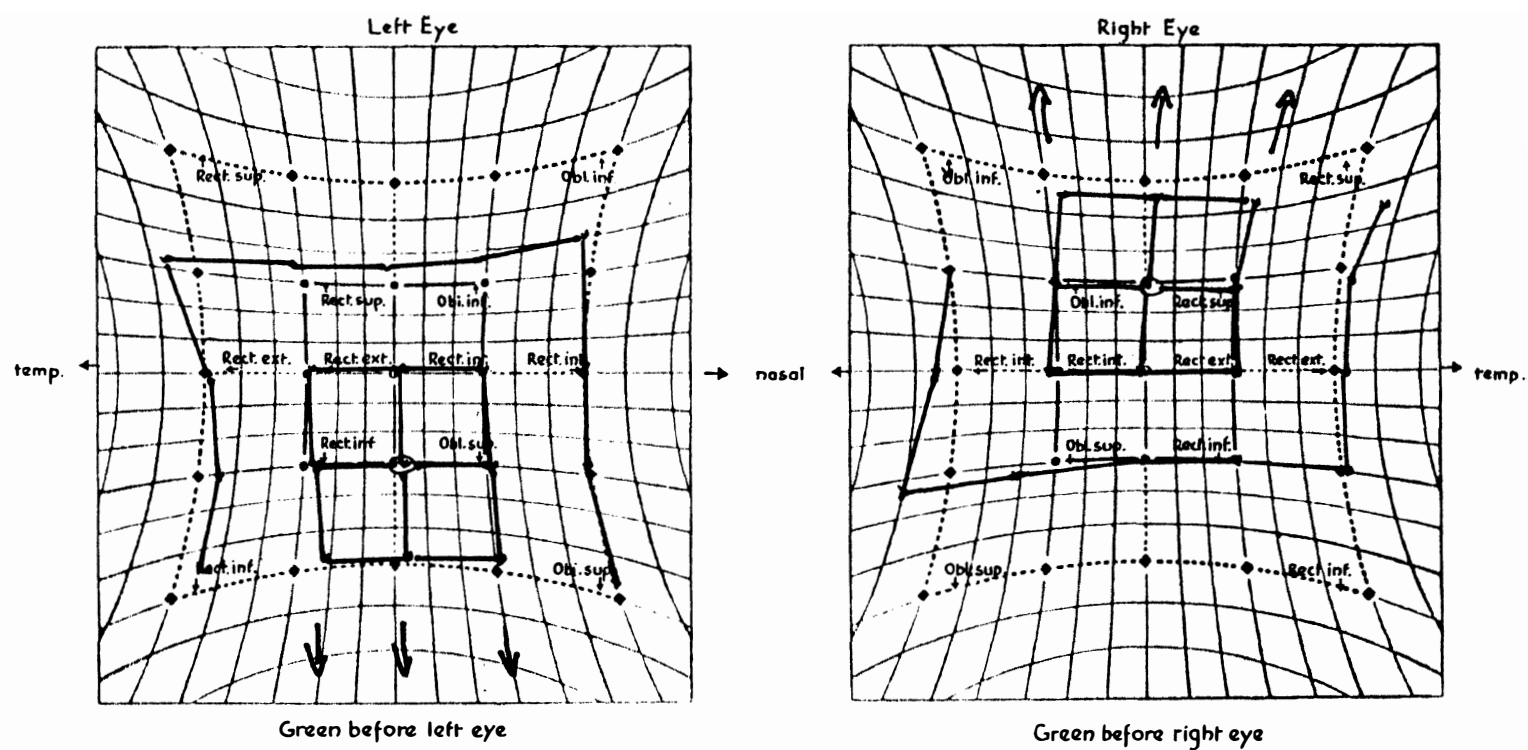

Fig. 1. Case 1. Hess chart following two doses of botulinum toxin for exposure keratitis.
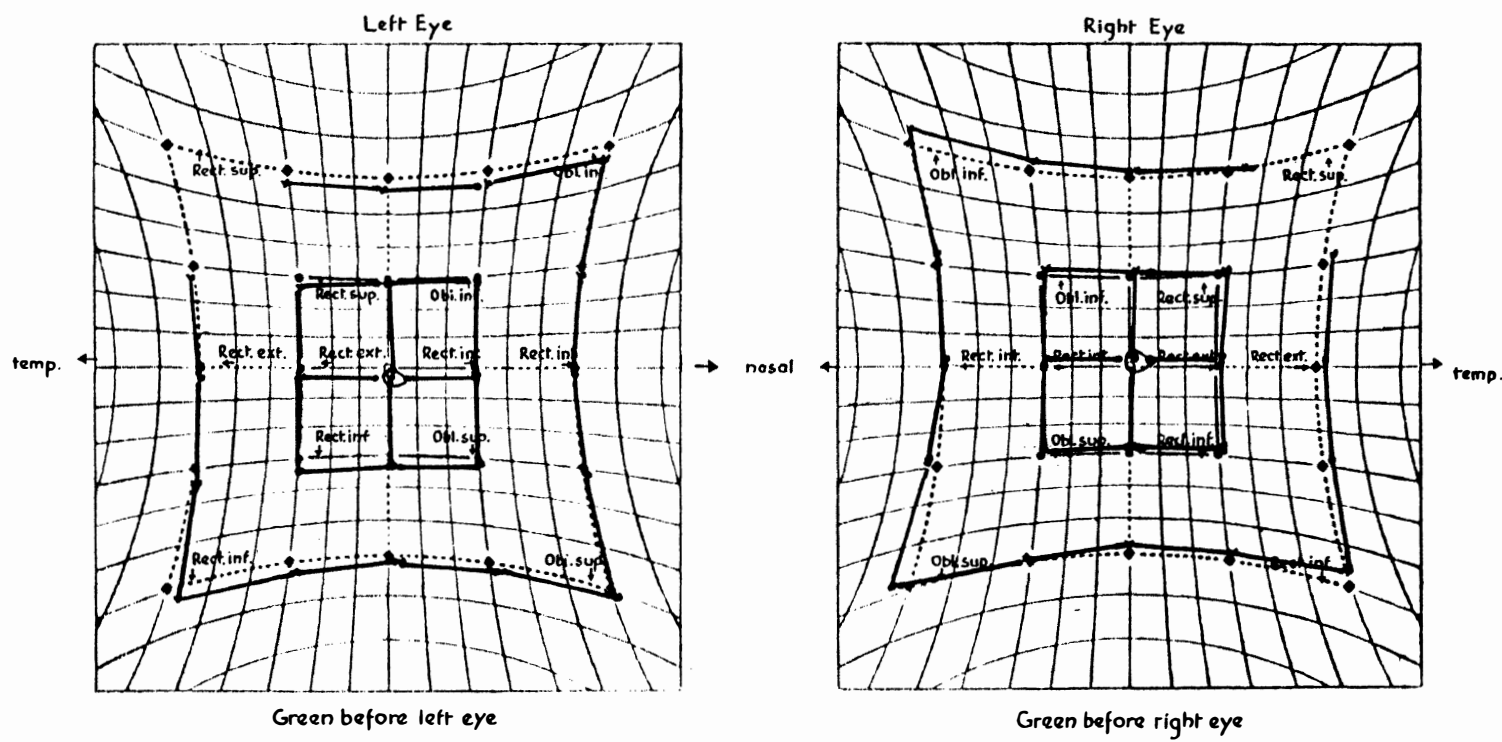

Fig. 2. Case 1. Hess chart following a further standard dose of botulinum toxin to the left inferior rectus.
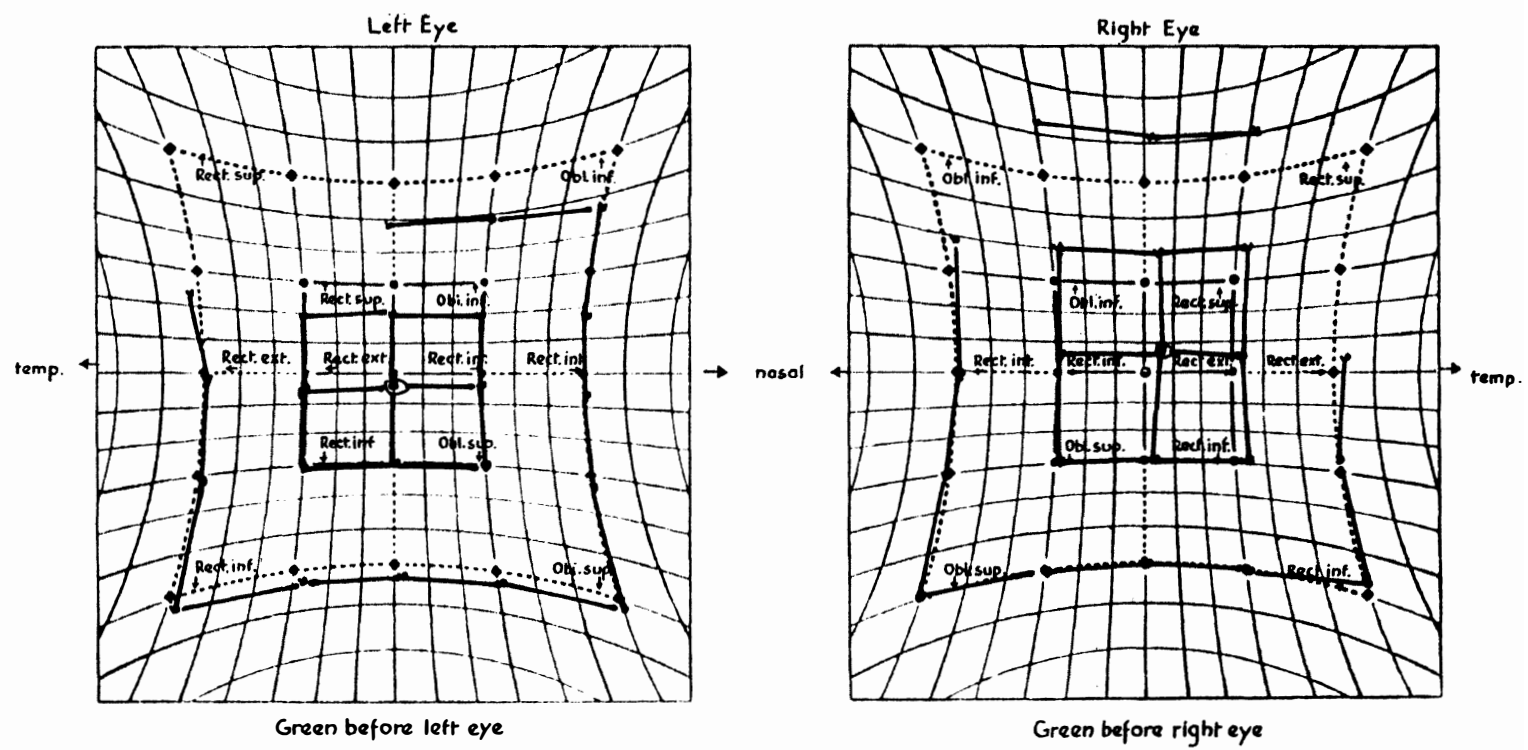

Fig. 3. Case 1. Hess chart following adjustable inferior rectus recession. 

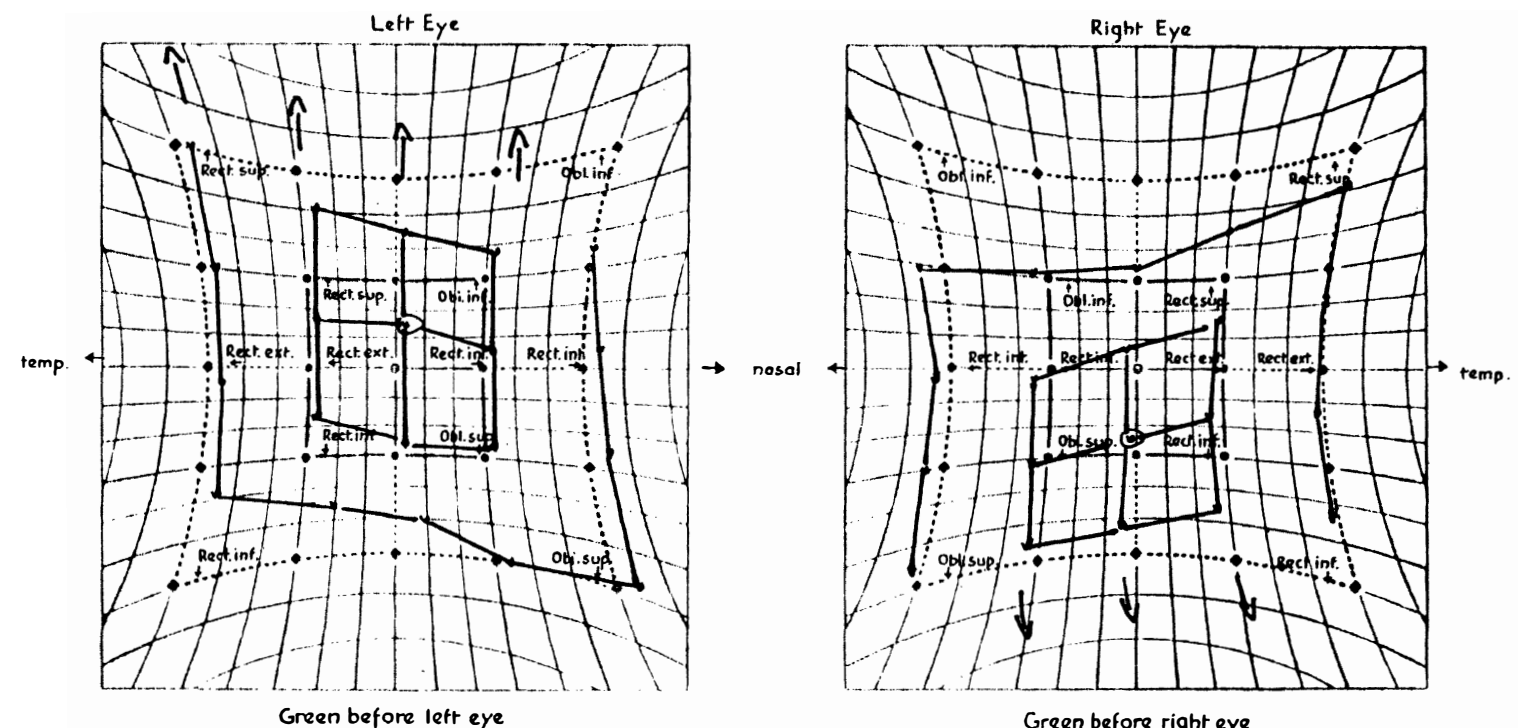

Fig. 4. Case 2. Hess chart following two doses of botulinum toxin for exposure keratitis.
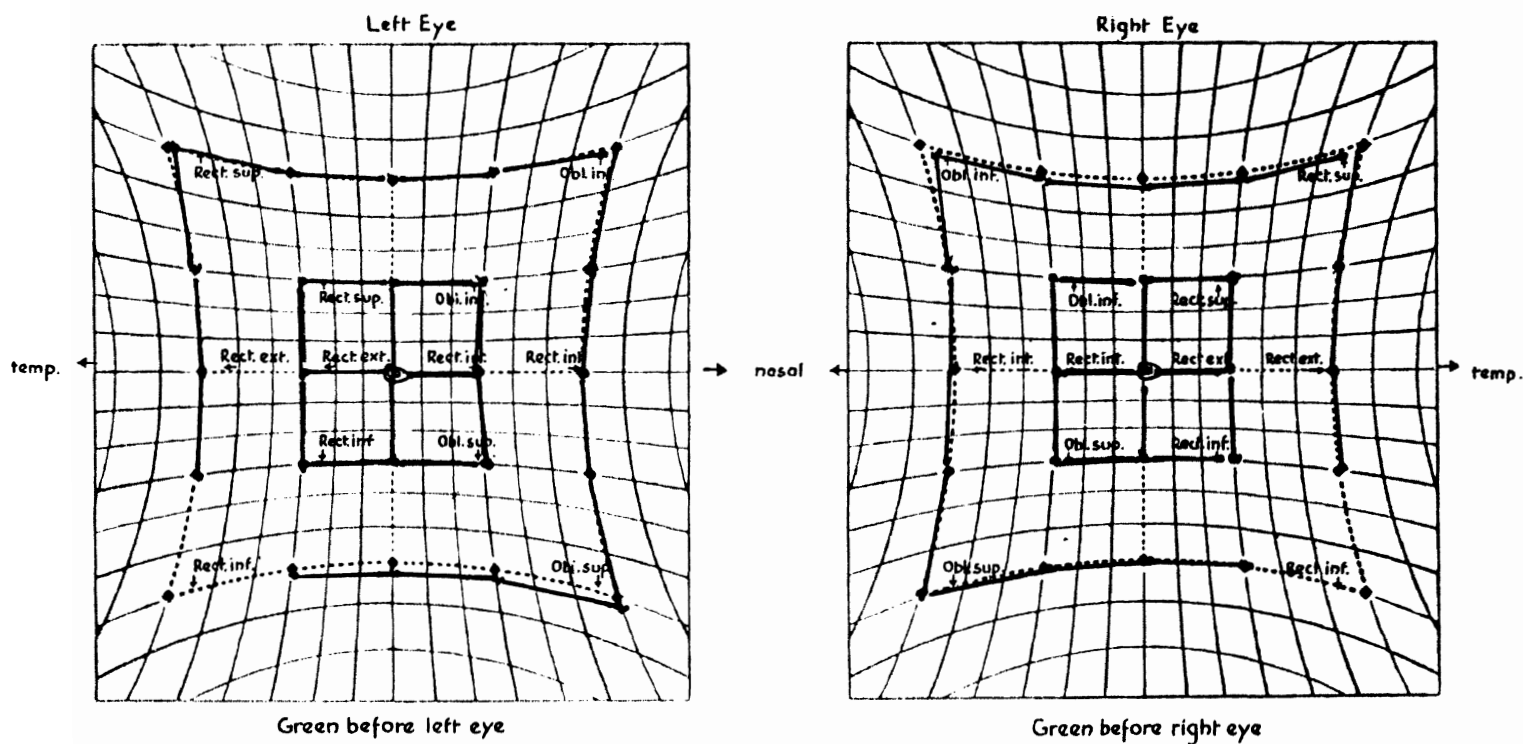

Fig. 5. Case 2. Hess chart following a Knapp procedure.
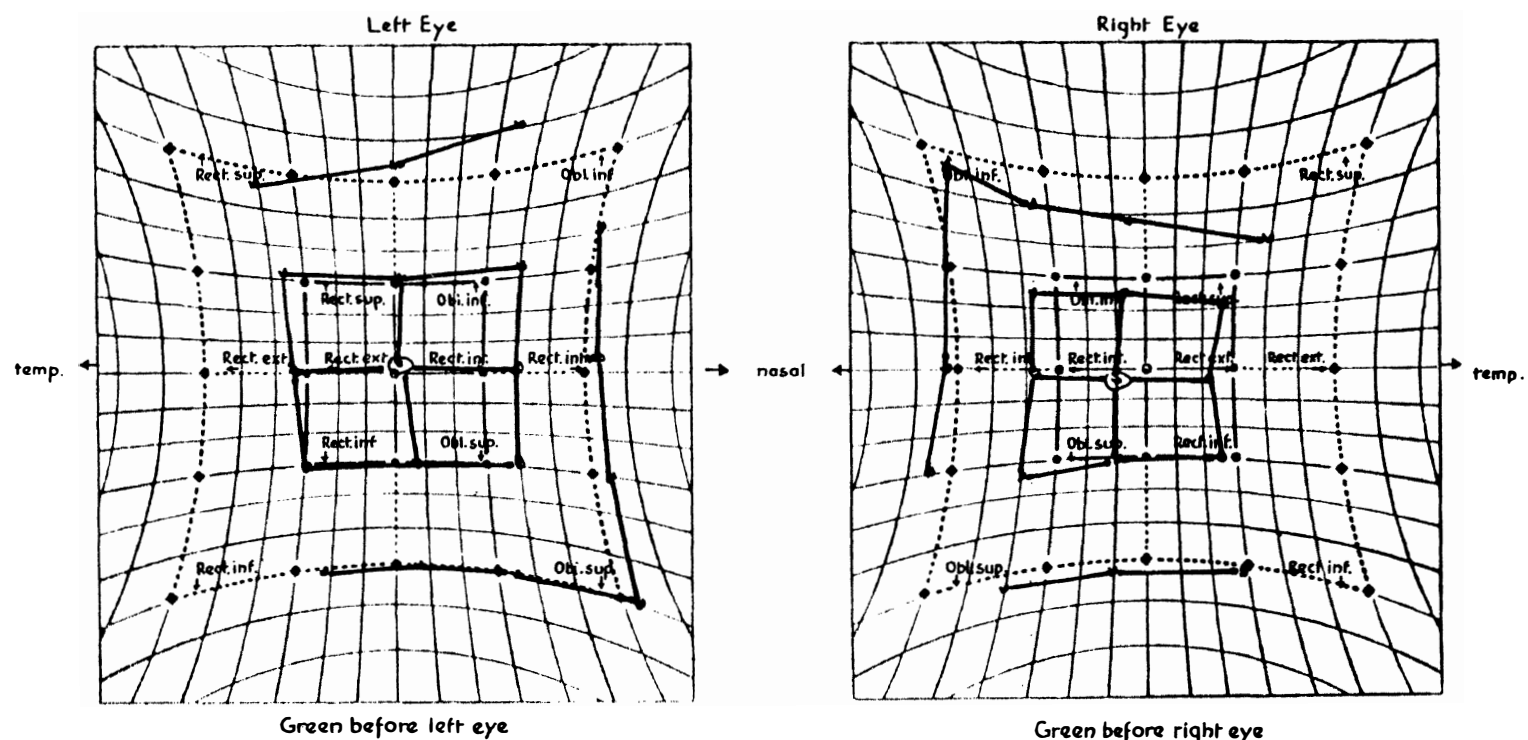

Fig. 6. Case 3. Hess chart following a right medial and right lateral rectus recession. 

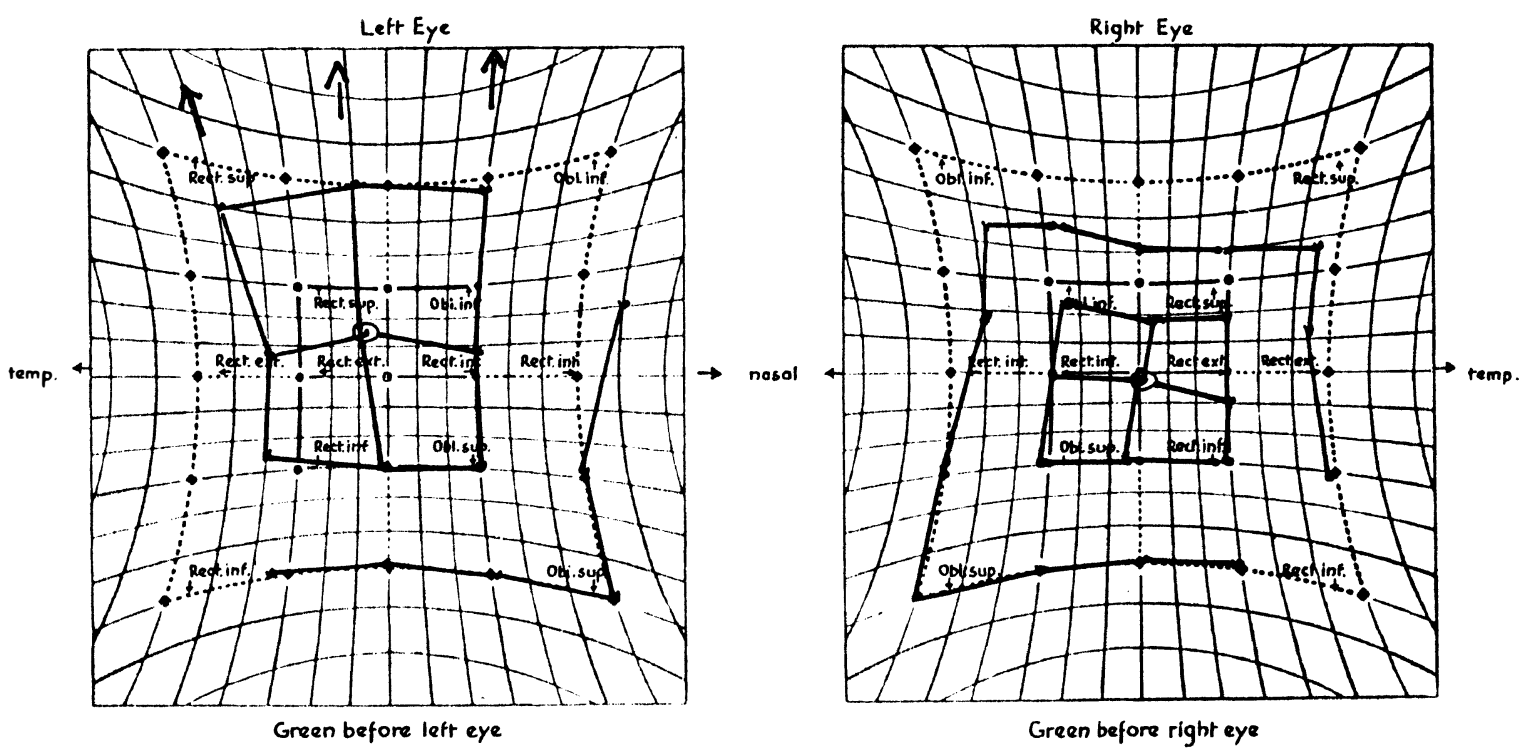

Fig. 7. Case 3. Hess chart following a single dose of botulinum toxin for exposure keratitis.
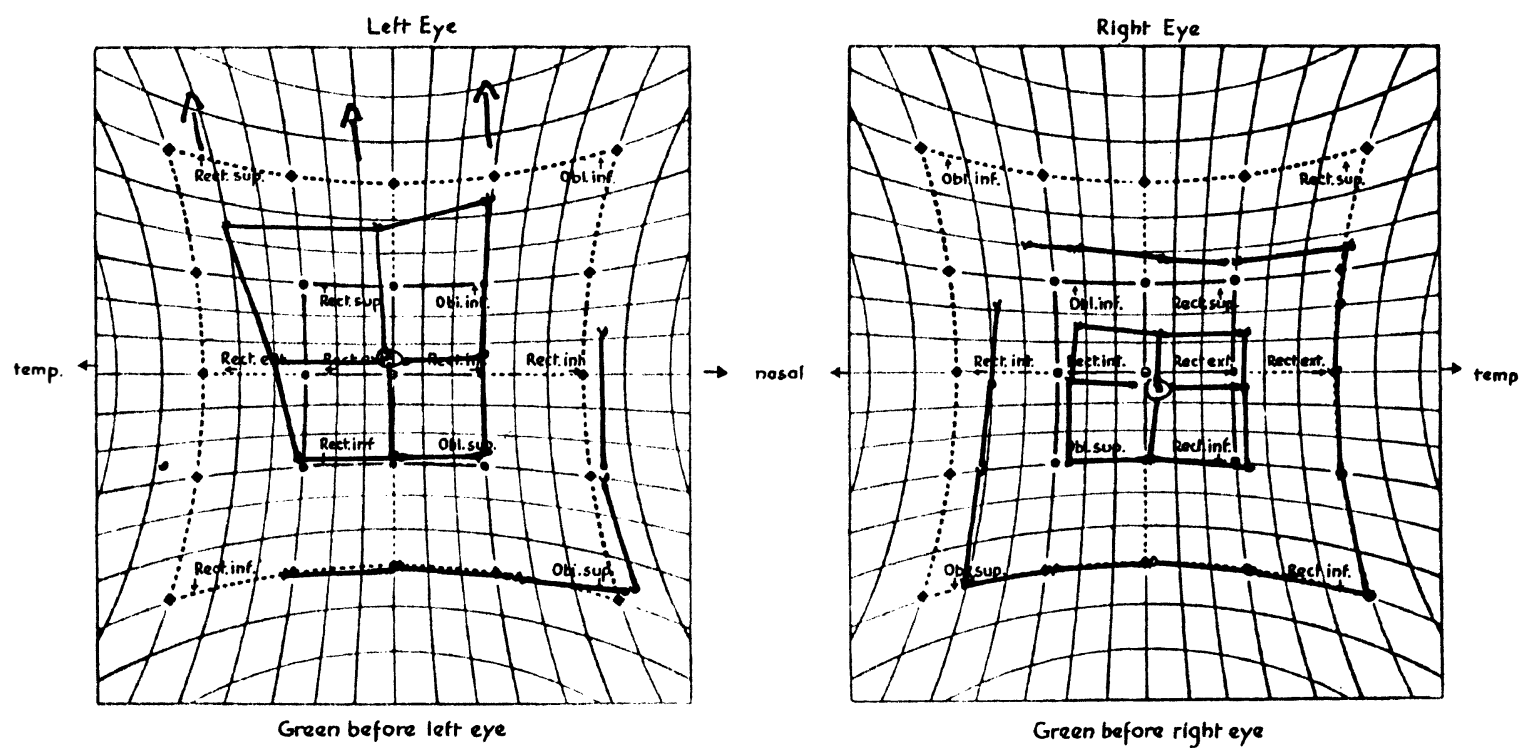

Fig. 8. Case 3. Hess chart following right inferior rectus recession and left inferior rectus Fadenisation.

to all positions of gaze (Fig. 5). The patient has been discharged from clinic.

\section{Case 3}

A 56-year-old man with known thyroid myopathy developed a right-sided esotropia. He underwent a right medial rectus recession and right lateral rectus resection. One month following surgery he was orthotropic in the primary position; however, he complained of diplopia on elevation. The Hess chart at this time shows bilateral restriction of elevation, more marked on the right side (Fig. 6).

One year following surgery the patient sustained a skull fracture following a fall. The fracture line extended into the parietal and occipital regions resulting in a lower motor neuron seventh nerve palsy which was followed by exposure keratitis. He was treated with a single dose of botulinum toxin to the right upper lid. Two months fol- lowing this he complained of vertical diplopia in the primary position and was recorded as having a 25 dioptre hypotropia in the primary position. A marked inferior rectus restriction compatible with thyroid myopathy can be seen (Fig. 7). Traction testing confirmed the restriction and the patient underwent a right inferior rectus recession and left inferior rectus Fadenisation. He still complains of diplopia in the primary position when not wearing a prism and the inferior rectus restriction persists (Fig. 8).

\section{DISCUSSION}

Botulinum toxin paralyses by preventing acetylcholine release from the motor end plate. ${ }^{2}$ The paralysis is temporary and recovery occurs due to the sprouting of new motor nerves and the formation of new motor end plates. ${ }^{3}$ The rate of recovery depends on the nature of the muscle fibre, fast or twitch fibres recovering at a slower rate. ${ }^{4}$ Adams $e t$ al. ${ }^{1}$ reported that the ptosis lasts for a mean of 2-3 weeks 
and recovers in a mean of 8.1 weeks. They reported that in $80 \%$ of cases there was a weakness of the superior rectus which lasted for a mean of 6 weeks although in one case full recovery was not complete until 5 months. We have identified three patients in whom the superior rectus weakness was permanent.

We do not necessarily propose that the permanent hypotropias induced by botulinum toxin are due to an irreversible myopathic effect. When used on the rectus muscles full function is restored with time, although there is some evidence that with chronic use botulinum may lead to long-term alignment changes. The patients recorded here received a maximum of only two doses. We propose two possible mechanisms for the persisting hypotropias. Firstly a period of occlusion in patients with a pre-existing latent deviation might result in a breakdown in fusion. Secondly, following paralysis of the superior rectus muscle there may be a co-contracture of the ipsilateral antagonist muscle further contributing to fusional breakdown. We do not suggest that all patients are likely to be susceptible to this complication, rather that patients with pre-existing latent vertical deviations are at risk. All three patients had good evidence of a pre-existing latent deviation. In case 1 there is a fair degree of concomitance suggesting that the deviation was long-standing (Fig. 1). In case 2 there was a superior oblique overaction and the patient could intermittently overcome the 25 dioptre hypotropia, suggesting that the elevated vertical fusion range was long-standing. In case 3 thyroid myopathy had resulted in bilateral inferior rectus restriction prior to treatment with botulinum toxin.

With hindsight and detailed orthoptic assessment these patients could all have been considered at risk. We estimate that the number of patients receiving this treatment at our hospital between the first and the last of the cases reported was 200. The incidence could therefore be as high as $1.5 \%$. There may, however, be many patients with chronic ocular conditions and poor vision in whom the deviations are unnoticed, thus possibly making the frequency higher.

In conclusion, botulinum-induced protective ptosis may lead to irreversible ocular imbalance, especially in those patients with latent deviations. Patients should be aware of this small risk and its implications when consenting to the BTXAP procedure.

Key words: Botulinum toxin A, Fusion, Hypotropia, Ipsilateral antagonist, Ptosis.

\section{REFERENCES}

1. Adams GGW, Kirkness CM, Lee JP. Botulinum toxin A induced protective ptosis. Eye 1987;1:603-8.

2. Burgen ASV, Dickens F, Zatman LJ. The action of botulinum toxin on the neuro-muscular junction. J Physiol (Lond) 1949; 109:10-24.

3. Duchen LW, Strich SJ. The effects of botulinum toxin on the pattern of innervation of skeletal muscle in the mouse. Q J Exp Physiol 1968;53:84-9.

4. Duchen LW. Changes in the electron microscopic structure of slow and fast skeletal muscle fibres in the mouse after local injection of botulinum toxin. J Neurol Sci 1971;14:61-74. 